\title{
Oral Profile of Patient Effected by Chikungunya Virus: A Case Report
}

$\begin{array}{ll}\text { Ayesha Iqbal }^{1} & \text { BDS, MSc } \\ \text { Sarah Hanif }^{2} & \text { BDS } \\ \text { Suraiya Hirani }^{3} & \text { BDS, MSc } \\ \text { Faiza Arif }^{4} & \text { BDS } \\ \text { Sarah Hussain }^{5} & \text { BDS }\end{array}$

Chikungunya is a febrile viral illness transmitted widely to human by the infected bite of mosquito families of Aedes aegypti and Aedes albopictus. Epidemiologically, the outbreaks of chikungunya virus was extracted from Southeast Asia and Africa. A variety of symptoms has been observed including onset of fever, joint pain, rashes and mucocutaneous manifestations.A female patient visited our private clinic with pain, trismus and with burning sensation while eating. The history and clinical examination was not enough to give clear diagnostic picture so a complete blood count including malarial parasite was requested. The lab investigations together with the signs and symptoms were strong to suggest that the female was suffering with chikungunya. The patient was prescribed with a good dose of ibuprofen, mouthwashes and was rescheduled for follow up. Chikungunya is a painful progressive disease of joints with of 7-12 days incubation period. The oral cavity is also targeted with limited mouth opening, gingivitis and ulcers depending on the severity of this condition.

KEY WORDS: Ooral manifestations, chikungunya virus, Fever

HOW TO CITE: Iqbal A, Hanif S, Hirani S, Arif F, Hussain S. Oral profile of patient effected by chikungunya virus: A case report. J Pak Dent Assoc 2019;28(3):149-152.

DOI: https://doi.org/10.25301/JPDA.283.149

Received: 26 December 2018, Accepted: 26 May 2019

\section{INTRODUCTION}

$\mathrm{T}$ he virus Chikungunya is a single-stranded positive sense RNA virus from both Togaviriade and genus Alphavirus. The mode of transmission is predominantly via an infected mosquito Aedes yet it is also transmitted vertically (mother to fetus), in addition transmission is also by cross infection through an infected person. A.aegypti and A.albopictus mosquitoes are the main vectors for transmission found dominantly in the areas of Asia and Africa where most of the cases were reported. ${ }^{1}$ The word chikungunya in some east African language means "to walk bent over" which is related to the symptom of severe arthralgia associated with the disease. The first significant outbreaks of the disease were first reported in 2004 in Kenya after which the large epidemic was reported in the areas of Indian Ocean and south East Asia. ${ }^{2}$ Chikungunya infection

1. Senior Lecturer, Department of Oral Pathology, Sir Syed College of Medical Scienes

2. Lecturer, Department of Oral Biology, Sir Syed College of Medical Sciences.

3. Senior Lecturer, Department of Oral Biology, Sir Syed College of Medical Sciences.

4. Lecturer, Department of Oral Biology, Sir Syed College of Medical Sciences.

5. Lecturer, Department of Community Dentistry, Sir Syed College of Medical Sciences.

Corresponding author: "Dr. Ayesha Iqbal” < ayeshaiqbal690@yahoo.com > can manifest as an emergency sometimes and it was first reported as a clinical emergency requiring the need of intensive care unit (ICU) Reunion Island epidemic in 2006. Areas such as Comoros, Mauritius, Seychelles, Madagascar and Reunion were largely affected. In Reunion millions of cases were reported with a death rate of 1 per 1000 clinical cases. Regardless of the death rate the morbidity rates were quite high. Recently in the areas of India around 1.3 million cases were reported. Further increase in the number of case reported is expected this year as well. The disease has epidemic and inter epidemic periods. During the epidemic period humans are the main reservoirs, while during inter epidemic periods, vertebrates such as birds, rodents and other small mammals are involved. A bacterium Wolbachia, naturally present in $60 \%$ of insect species which is known to have vector control capacity, as it is an intracellular resident of many viruses including Chikungunya virus. ${ }^{3}$ The ability of Wolbachia to alter host physiology, its pathogen blocking phenotype, has provided a benefit to its host limiting insect population. Chikungunya virus usually hits draught affected areas where there is no proper water storage system, this promotes mosquitos to breed in the stored water. Furthermore 
the mosquitoes responsible for the spread of A. aegypti and A. albopictus are capable of surviving at a high temperature range. This was the reason why most of the tropical regions were affected. These mosquitoes are very resistant to insecticides which makes the disease control further difficult. ${ }^{2}$

The disease is divided into acute and post-acute phase. In acute phase the patient presents with extreme fatigue, arthralgia and myalgia along with numerous muco-cutaneous manifestations which include blisters, erythema nodosum, exfoliative dermatitis, photosensitivity and ulcers ${ }^{4}$ whereas the post-acute phase begins 3 weeks after the acute phase the patient becomes asymptomatic showing transitory improvement but with persistent muscle and joint pain. ${ }^{5}$ If arthralgia persists for more than 3 months the disease enters chronic phase in which the patient may present with other clinical manifestations for months or even years. Results of scientific work demonstrates that the symptoms of adults are different from children. High grade fever for 4-7 days, vomiting, diarrhea, eye infection and loss of appetite are the symptoms variably in children whereas in adults all these symptoms are commonly seen along with headaches, body aches and joint pain which can be severe and disabling.

Rare complications include encephalopathy, myocarditis and hepatitis and multi organ failure. Patients with low immunity due to preexisting systemic disease such as diabetes and other cardiovascular and respiratory diseases are at a high risk of getting these complications. Neonates and children also presents complication. Uveitis and retinitis are also recently diagnosed complications. ${ }^{6}$

Clinical presentation, epidemiology and laboratory tests are required for the diagnosis of chikungunya. ${ }^{7}$ Differential diagnosis should comprise of other mosquito borne diseases (such as dengue and malaria) through proper laboratory tests and clinical examination. Laboratory tests are based on viral RNA identification through reverse transcription and real time PCR by detection of IgM and IgG antibodies through serological tests using ELISA or rapid immunochromatographic tests. The RT-PCR based technique provides a rapid and sensitive result but only on the seventh day of onset of symptoms. ${ }^{8}$

Absolute rest, maintain hydration and prescription of analgesics such as paracetamol to control muscular and joint pain was advised to treat the patient symptomatically. ${ }^{4}$ Non-steroidal anti-inflammatory drugs are only used once dengue is excluded and in cases of severe arthralgia. Studies have been carried out to produce antivirals and vaccines to control the disease. A live, attenuated vaccine was introduced but was not considered safe as arthralgia was reported in the few cases after the administration of the vaccine. Another safer and effective technique is also introduced using a combination of chimeric alphaviruses along with chikungunya virus structural protein genes. These vaccines succeeded in generating an antibody response to the chikungunya virus however residual ability to infect potential mosquito makes it still a question to the introduction of the vaccine. Through recent epidemics, a lot of information has been gained regarding the virus however no such vaccine has been introduced to control the spread. Measures should be taken to educate people about the disease and its spread in the epidemic areas to the travelers along with introducing strategies to control mosquito bites The failure of the vector control strategy to reduce the risk of the disease and lack of commercial vaccine has increased the need for more options to prevent the spread of this disease. ${ }^{9}$

\section{CASE REPORT}

A 29-year-old female patient, reported with complaint of mild trismus and high fever $\left(>39^{\circ} \mathrm{C}\right)$, to my private practice. History revealed that she was suffering from fever and joint pain since last 7days, morning stiffness of all joints which aggravated within few hours making her unable to come the clinic on her own.

The patient was found exhausted and in severe pain. Intra oral examination revealed mucosal involvement with cheilitis, multiple small aphthae, erosions with severe pain, generalized gingivitis and burning sensation while speaking and swallowing compromised oral hygiene inability to chew food and loss of taste.

On examination patient was found to have trismus and facial swelling. We prescribed her mouth gels, mouth washes, some exercises to overcome trismus and advised lab investigation to rule out chikungunya. Furthermore the patient OPG revealed limited mouth opening, rest of dentition was normal. The patient was re scheduled after two days

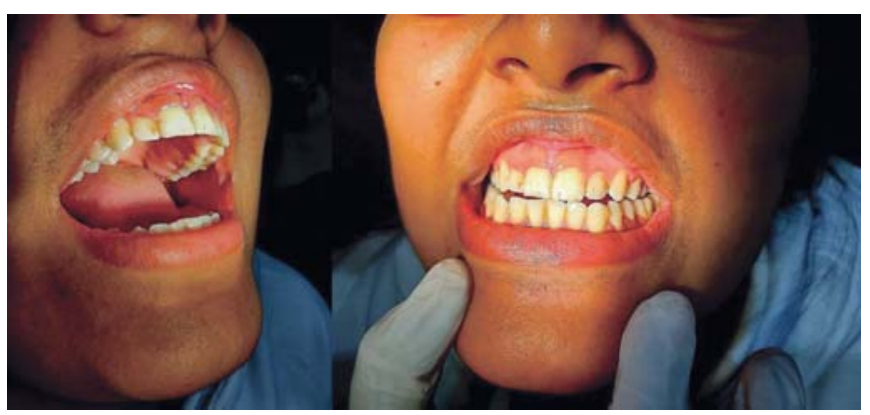

Fig 1 \& 2: Showing intra oral clinical pictures of patient suffering from chikungunya with limited mouth opening and ginigivitis.

with her reports but the patient sent the reports with her husband as she had severe joint pain and could not travel. According to the hematological lab report the total white blood cell count was $2.7 \times 10^{\wedge} 9 / \mathrm{L}$ and the platelet count was 


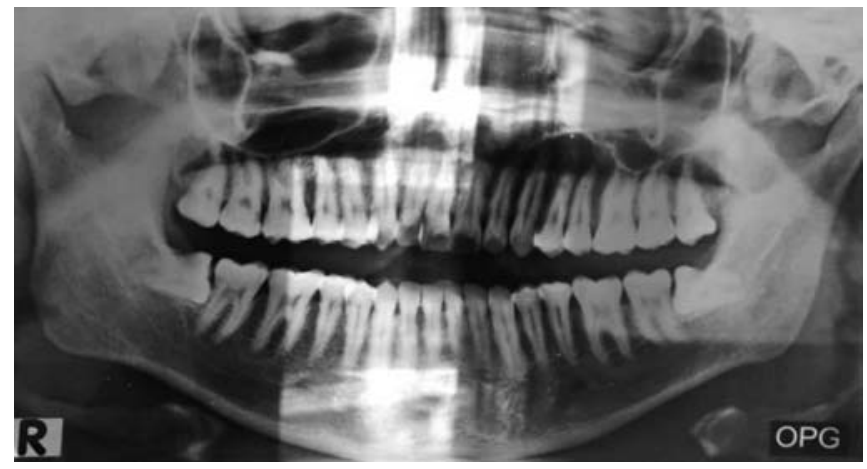

Fig 3: Showing OPG of same patient showing limited mouth opening

$14 \times 10^{\wedge} 9 / \mathrm{L}$. The lab reports and patient's history suggested leucopenia and thrombocytopenia along with joint pain which is an absolute signs of chikungunya. By looking at the finding we referred the patient to General physician for further investigations and treatment immediately. For our concern, our team did the follow up by contacting her and assured that the patient is visiting the referred physician.

\section{DISCUSSION}

Chikungunya fever is an acute delirious disease infected by an arthropod-borne alphavirus. This disease is principally transmitted by the virus to humans via the infected mosquito bite of Aedes species. A.albopictus is referred to as "Asian tiger mosquito" acting as the main vector for the spread and pathogenicity especially in area of Asia. During 1950s CHIKV was initially documented as a human pathogen in Africa, and subsequently then, cases have been acknowledged in multiple affected countries in Africa and Asia. In 2004, in Kenya CHIKV re-emerged and consequently had a blowout eastward affecting millions of people which reported cases in around the Indian Ocean. Later in 2007, this was then reported in Europe with massive outbreak in Italy which then move to Europe, Western Hemisphere and Australia. Clinical presentation of chikungunya can be confused with that of dengue as both presents with sever joint pain. However, symptoms such as gastrointestinal hemorrhage and shock were mainly presented by patients suffering from dengue, furthermore the onset of symptoms is more abrupt in patients with chikungunya than that of dengue. ${ }^{10}$

The spread of Chikungunya virus was noticed with some focal clinical symptoms manifesting fever (99.6\%), muscle pain $(97.7 \%)$, headache $(84.1 \%)$ and joint pain (99.2\%). Since the beginning of the outbreak in Reunion there was no reported case of direct death due to chikungunya infection. The mortality rate associated with chikungunya virus is quite low (case fatality rate $0.1 \%-5 \%$ ) as compared to the other similar diseases such as dengue and zika, however morbidity rates are higher due to persistent chronic arthritis. In immunocompromised patients and those undergoing transplants while having chikungunya, are seen to have comparatively less incubation period than those having dengue.

This disease according to the surveillance system is labelled as spatiotemporal trends and to identify early spread for optimize control and treatment measures. CHIKV has a low vectorial transmission and for that it is not reported in high altitudes areas. ${ }^{11}$ CHIKV disease represent with a range of multiple systemic complications such as ophthalmological, neurological, myocardial, oral and dermatological disorders. Chikungunya fever has emerged as a worldwide disease.

\section{CONCLUSION}

A challenging role as a dental professional is to rectify the oral status of the current infection which plays a major role in assessing the diagnosis and ultimately the treatment of it. In severe cases chikungunya virus is found rapidly affecting various organs in patients. As in this case the patient was found in acute phase so organ analysis was not done and the findings of the patient reported were high grade fever, trismus, joint pain, morning stiffness and intra oral ulcers. The laboratory reports revealed leucopenia therefore we referred the patient to General physician for symptomatic treatment.

\section{CONFLICT OF INTEREST}

None declared

\section{REFERENCES}

1. Burt FJ, Rolph MS, Rulli NE, Mahalingam S, Heise MT. Chikungunya: a re-emerging virus. The Lancet. 2012;379(9816):66271.

https://doi.org/10.1016/S0140-6736(11)60281-X

2. Control CfD, Prevention. $\mathrm{CDC}$ health information for international travel 2014: The yellow book: Oxford University Press; 2013.

3. Pham P, Williams L, Obot U, Padilla L, Aung M, Akinyemiju T, et al. Epidemiology of Chikungunya fever outbreak in Western Jamaica during July- December 2014. Res Reports Trop Med. 2016;55:7-16.

https://doi.org/10.2147/RRTM.S122032

4. da Cunha RV, Trinta KS. Chikungunya virus: clinical aspects and treatment-A Review. Memórias do Instituto Oswaldo Cruz.

2017;112:523-31.

https://doi.org/10.1590/0074-02760170044 
5. Simmons G, Brès V, Lu K, Liss NM, Brambilla DJ, Ryff KR, et al. High incidence of chikungunya virus and frequency of viremic blood donations during epidemic, Puerto Rico, USA, 2014. Emerg infect dis. 2016;22:1221-28.

https://doi.org/10.3201/eid2207.160116

6. Al Mahdy A, Jamal M, Kinoshita H, Hossan T. Chikungunya virus outbreak-a threat to global public health including Bangladesh. Bangl J Med Sci. 2018;17:183-84.

https://doi.org/10.3329/bjms.v17i2.35868

7. Mahaver M, Sharma N, Khinchi M, Khan MS. A Brief Review on Chikungunya. Asian J Pharmaceut Res Develop. 2017:1-7.

8. van Aalst M, Nelen CM, Goorhuis A, Stijnis C, Grobusch MP. Long-term sequelae of chikungunya virus disease: A systematic review. Travel med infect dis. 2017;15:8-22.

https://doi.org/10.1016/j.tmaid.2017.01.004
9. Powers AM. Vaccine and therapeutic options to control Chikungunya virus. Clinic microbi reviews. 2018;31:e00104-16. https://doi.org/10.1128/CMR.00104-16

10. Gupta A, Juneja D, Singh O, Garg SK, Arora V, Deepak D. Clinical profile, intensive care unit course, and outcome of patients admitted in intensive care unit with chikungunya. Indian J criti care med. 2018;22:5.

https://doi.org/10.4103/ijccm.IJCCM 33617

11. Lee N, Wong CK, Lam WY, Wong A, Lim W, Lam CW, et al. Chikungunya fever, Hong Kong. Emerg infect dis. 2006;12:179092.

https://doi.org/10.3201/eid1211.060574 